\title{
Mitra Azar
}

\section{POV-MATTER, CINEMATIC POV AND ALGORITHMIC POV BETWEEN AFFECTS AND UMWELTEN}

\begin{abstract}
The essay refers to affect theory as a conceptual toolbox to draw a genealogy of POV (Point of View) that goes from the formation of the first organic POV to the reinvention of POV by the cinematic apparatus up to the latest development of algorithmic POV in machine vision and Al. The essay engages with Bergson's conviction that there's no perception without affection, and tests it against a phenomenological, cinematic and machinic notion of POV. To do so, the essay introduces what the German biologist Jacob von Uexküll has called Umwelt - the ecological niche emerging from the affordances between organisms, space, and (when applicable) technology. Furthermore, fundamental categories of both phenomenology and psycho-analysis are put at work in relation to cinematic POV and to the algorithmic POV produced by Generative Adversarial Networks (GANs), which seems to re-invent the relationship between seeing/seen (Merleau-Ponty) and eye/gaze (Lacan). This re-invention confirms the category of Umwelt and affect as markers for understanding the transformation between a phenomenological, cinematic and algorithmic notion of POV.
\end{abstract}

\footnotetext{
APRJA Volume 8, Issue 1, 2019

ISSN 2245-7755
}

\section{CC license: 'Attribution-NonCommercial-ShareAlike'.}


Could a machine think?

Could it be in pain?

(Wittgenstein, Philosophical Grammar)

\section{POV, affects and Umwelten between phe- nomenology, cinema and machine vision}

In this essay, I propose affect theory as the conceptual framework for analyzing the various regimes of visibility related to the formation of different forms of 'Point of View' (POV). The notion of POV is here approached as a phenomenological feature that in its fundamental understanding goes beyond the visual connotation it has been usually associated with, and can be defined as 'orientation' tout court. In this sense, matter is oriented despite the organic/inorganic divide, and can be defined as POV-matter, once framed by the concept of orientation. Orientation manifests already at an inorganic level, right down to the spinning of particles inside atoms that function independently from organic POVs and associated affects. POV-matter takes the form of inorganic POVmatter and organic POV-matter, and the notion of affect is presented as the conceptual tool to understand the differences between the two.

What happens to inorganic POV-matter since the formation of the first organic POVmatter emerging from the pre-biotic soup?[1] There, the first proto-stable organic forms of life produce both an orientation and a gap appearing between the immediate action/reaction schema characterizing instead inorganic POV-matter - as demonstrated by Gilles Deleuze is his first book on cinema, Cinéma 1: The Movement-Image. The notion of affect as 'gap' discriminates between organic and inorganic POV-matter. There exists, in other words, a form of inorganic POV-matter characterized by orientation but not by the gap associated with orientation when orientation relates to an organic living agent.

Building from these first considerations, the essay asks what happens to affect when POV becomes associated to more complex forms of life equipped with a visual sensing apparatus, such as in the case of human beings. Furthermore, what happens to affect when POV becomes technological and associated to a visual and technological apparatus? Although inorganic POV-matter lacks an organic form of perception and affect, human beings have been able to turn inorganic POV-matter into a technological apparatus capable of harnessing both perception (and by doing so the organic POV-matter associated to it), and the affective gap itself. The technological apparatus can be referred to as a POV-apparatus because it is constituted by POV technologies of vision, which means technologies designed to harness phenomenological POV. Via analog POV technologies of vision such as painting, photography and cinema, the POV-apparatus attempts to harness affection via perception. Via what Mark B. N. Hansen calls ' $21^{\text {st }}$ century media' (266), POV-apparatus attempts to harness affection directly, and turns into a type of media which gains the capability of directly attacking the affective gap at the core of organic POV-matter, thus generating inorganic algorithmic POV capable of mimicking the functioning of organic POV.

In summary, the essay aims to investigate what happens to affect in relation to both the (pre)phenomenological and the technological (specifically cinematic and algorithmic) definitions of POV. By questioning the becoming of different forms of POV and their corresponding affects, the essay aims to engage with the French philosopher Henri Bergson's conviction that there's no 
perception without affection (Bergson 1776). To do so, the essay introduces what the German biologist Jacob von Uexküll has called Umwelt ("The new concept of Umwelt" 111-123), understood as the affordances generated by the interaction between organisms, space, and - when applicable, such as in the case of human beings - technology: "everything a subject perceives belongs to its perception world [Merkwelt], and everything it produces, to its effect world [Wirkwelt]. These two worlds, of perception and production of effects, form one closed unit, the environment [Umwelt]" (Uexküll, A Foray into the Worlds of Animals and Humans 42). Furthermore, the mutation of affect in relation to the transformation between a phenomenological, cinematic, and algorithmic notion of POV are finally approached in relation to the reversibility between seeing and seen (Merleau-Ponty, The Visible and the Invisible 130-155) and the relation between the notion of eye and gaze (Lacan 67-79). These fundamental categories of both phenomenology and psycho-analysis (Merleau-Ponty, The Visible and the Invisible 130-155; Lacan 6779 ), are put at work in relation to a specific form of algorithmic POV produced by Deep Convoluted Generative Adversarial Networks (DCGANs, or simply GANs), which seems to re-invent the relationship between seeing/ seen and eye/gaze. This re-invention invites rethinking the category of Umwelt and affect as markers for understanding the transformation between a phenomenological, cinematic and algorithmic notion of POV.

\section{From inorganic POV- matter to affects, organic POV-matter and Umwelten}

In a fundamental way, POV can be understood as orientation. After the Big Bang, fundamental blocks of matter organized themselves by producing orientations, technically referred to as 'spins' within electromagnetic fields.[2] In other words, despite the organic/inorganic divide, matter is always orientational: matter is directional, and can, hence, be characterized as 'POV-matter'. Inorganic POV-matter indicates that orientation manifests itself in the inorganic world, and only afterwards turns into the orientation of organic POVmatter. Organic POV-matter exists from the simplest organisms deprived of a visual sensing apparatus, but yet capable to orient via other senses (such as the 'tick'[3]) to more complex living beings developing the sense of vision, among them human beings. In contrast to inorganic POV-matter, organic POV-matter perceives. Thinking generally of matter as POV-matter is not trivial, because it allows us to not only think of orientation or POV as pivotal ontological and phenomenological concepts, but also and more importantly because it allows to re-articulate the agential relation between the organic and the inorganic. In this non-trivial sense, a POV is not something that comes exclusively with the human or with the technological. Rather, POV as the production of an orientation has cosmological origins which not only predates the appearance of human POV but also the appearance of the first form of simple organic POVs in the prebiotic soup.

What then happens to orientation when orientation becomes embedded into organic living being inhabiting a Umwelt, understood as the ecological niche of a living organism? If inorganic matter is, in a way, always 
inorganic POV-matter, Umwelt is in a way always POV-Umwelt, because it always refers to an oriented subjective experience (although not forcibly human). Every Umwelt "has its own spatial and temporal dimensions" (Uexküll, A Foray into the Worlds of Animals and Humans 49), and " [they] intersect in many ways without disturbing each other" (Uexküll, "The New Concept of Umwelt" 117). As Uexküll further explains: "every action [...] that consists of perception and operation imprints its meaning on the meaningless object and thereby makes it into a subject-related meaning-carrier in the respective Umwelt (subjective universe)" (Uexküll, "The Theory of Meaning" 31). Otherwise expressed, when (according to different evolutionary survival criteria) inorganic POV-matter develops into organic forms that orient themselves in space, the various forms of POV-matter produce their unique Umwelten - of which the organism's regime of visibility represent the visual counterpart. Orientation, or POV - in the form of a perceptive agent - produces Umwelt. At the same time, in turn, Umwelt produces POV. Furthermore, the formation of the organism's Umwelt is specifically related to the affective quality of organic POV-matter, because it is in the affective gap between action and reaction that an orientation is produced, and together with it the beginning of an Umwelt. In this sense, affect establishes itself as the inner engine of orientation, and orientation as the inner engine of Umwelt.

Inorganic POV-matter turns into the technological and cultural expression of complex organic POVs such as human beings, and becomes a cultural and technological product. In their technological instantiation, spins and fields of atoms' particles are geared towards the construction of technologies able to reproduce the regime of visibility correspondent to the Umwelt of a given organism. Inorganic technological POVs, attempt to mimic the functioning of organic POVs, and by doing so they manage to overlap and ultimately bridle human POVs and their Umwelten. Inorganic POV-matter becomes technological POV-matter, or POVapparatus. A POV-apparatus of analogue technologies (such as painting, photography and cinema) is a form of visual governmentality developed to ultimately harness humans' POV and subsume affection via perception. A digital or algorithmic POV-apparatus (such as the one enforced by artificial intelligence and GANs) bypasses perception, and operates at the affective gap to predict the emerging affections and related affordances of organic POVs within a given Umwelt.

\section{Organic POV-matter and affects}

In this section, I argue that organic POVmatter is defined as a form of orientation co-emerging with the affective gap during the formation of the first proto-stable organic forms of life. As mentioned earlier, according to Bergson, there is no perception without affection (17-76). In other words, there is an intrinsic relation between organic POV and affects. Bergson's statement is adopted by Deleuze, in Cinéma I: The Movement-Image, to categorize cinematic images: "The thing is the image as it is in itself, as it is related to all the other images to whose action it completely submits and on which it reacts immediately. But the perception of the thing is the same image related to another special image which frames it, and which only retains a partial action from it" (Deleuze, The Movement-Image 64).

From a universe composed by images that "act and react on all their facets and in all their parts" (Deleuze, The Movement-Image 61), Deleuze unfolds the image-perception to name a type of image which "only receive[s] 
actions on one facet or in certain parts and only execute reactions by and in other parts. [...] the image reflected by a living image is precisely what will be called perception" (Deleuze, The Movement-Image 62). In other words, image-perception is the image reflected by organic POV-matter. This "image reflected" coincides with the regime of visibility of a given Umwelt for a given organic POV-matter. Nevertheless, how does inorganic POV-matter turn into organic POV-matter?

Affect - understood as the production of a gap between action and reaction - is the catalyst for the production of affects and for the formation of organic POV-matter(s) and their respective Umwelten. Umwelt is here understood as the selective interaction between organic and inorganic POV-matter (e.g. a species and their environments, also considered in their inorganic composition). Orientation is what emerges together with affects from the gap, producing both a POV and a Umwelt. In the affective gap between action and reaction POV-matter orients itself according to the elaboration of the input (action) from the surrounding environment and towards an output or reaction - this circuit being what Uexküll refers to when he says that "everything a subject perceives belongs to its perception world [Merkwelt], and everything it produces, to its effect world [Wirkwelt]. These two worlds, of perception and production of effects, form one closed unit, the environment [Umwelt]" (Uexküll, A Foray into the Worlds of Animals and Humans 42).

Orientation is the fundamental resolution of organic POV-matter(s) to their specific Umwelten - and emerges from the affective gap at the foundation of organic POV-matters to harness the affordances appearing between organic POV-matter(s) and their Umwelten. Affects fill the seemingly empty gap between action and reaction defining the emergency of organic POV-matter: "the interval is not merely defined by the specialization of the two limits facets, perceptive and active. There's an in-between. Affection is what occupies the interval, what occupies it without filling it in or filling it up" (Deleuze, The Image-Movement 65). This is how the difference between inorganic and organic POV-matter comes into being. Organic POV produces a gap between the continuous action-reaction characterizing the functioning of inorganic POV-matter. The gap is where the action-reaction circuit is interrupted. "Even at the level of the most elementary living beings one would have to imagine micro-intervals. Smaller and smaller intervals between more and more rapid movements" (Deleuze, The Movement-Image 71).

Once reduced to its essence, the affective gap can be intended as the figure for the coincidence between organic POV-matter and inorganic POV-matter, or, for simplicity, between subject and object. This is what happens, for instance, when the subject is automatically driven to bring attention to their body - such as in the case of hunger. Hunger happens in the gap between action (the action of the environment on the subject in the form of the presence of food) and reaction (the movement towards food by the subject) because of a moment of self-perception during which the subject becomes the object of their own attention, before enacting the appropriate reaction. Self-perception sprouts from the affective gap, short-circuiting the relation subject/object. This is interesting because cinematic POV produces something similar by perceptively overlapping the subject (audience/actor/director) with the object (camera/screen). By doing so, cinematic POV manages to access the affective gap at the core of organic POV-matter or phenomenological POVs. To put it simply, the functioning of cinematic POV mimics the way the affective gap functions at the level of phenomenological POV, and subsumes 
the affective gap via perception. Generally speaking, analogue POV technologies of vision such as painting, cinema and photography harness organic POV and the affective gap on the side of perception, aiming at shrinking the distance between technological and organic POV. Because of movement, the cinematic figure of POV operates this shrinking flawlessly and in doing so manages to short-circuit subject and object by giving to the audience the illusionary (self-) perception of breaking through the screen and of moving inside of it on behalf of the character. As a consequence, cinematic POV manages to harness the affective gap via the subject's activity of self-perception which emanates from it. Digital and algorithmic POV technologies in the form of $\mathrm{Al}$ and machine sensing - what Hansen refers to as $21^{\text {st }}$ century media - instead, attempt to harness organic POV and perception directly on the side of affection. Before looking at the role of the 'gap' in relation to machinic POVs, I will now discuss the form that inorganic POV-matter takes once it becomes cinematic apparatus, and more specifically, cinematic POV.

\section{POV in cinema and phe- nomenology: Reversibility between seeing/seen and split between eye/gaze}

In the field of cinema, POV is an acronym which refers to a type of image that allows the viewer to see what the character sees from the character's perspective (or orientation) (Braningan 55). POV cinematic images simulate the movement of an actor within a space, creating a sense of continuity between viewers and what is viewed, as if viewers are 'embodied' in the images they're looking at. In this sense, cinematic POV images generate the seamless overlapping between camera, actor's body and spectator's body, thus producing a form of seamless overlapping between the human and the technological. If technology and human have been overlapping since 'hominization',[4] with cinematic POV the overlapping is seamless because of the capacity of cinema to reproduce movement, a quality other medium before cinema couldn't attend to. Cinematic POV articulates the relation between the spectator's POV intended as the phenomenological orientation produced by an embodied human agent in a physical space and the regime of visibility produced by the cinematic machine. The very collapse and overlapping between the embodied agent's POV and the regime of visibility generated by the cinematic machine is the main feature of the cinematic technics of POV. Cinematic POV harnesses the embodied POV on the side of perception, and aims at shrinking the distance between technology and body, or between object and subject.

The possibility of generating the seamless overlapping between camera, actor's body and spectator's POV gives to cinematic POV the ability to intervene into the affective gap at the core of organic POV by re-articulating the relation between the phenomenological categories of seeing/seen and eye/ gaze. From a phenomenological perspective, one of the main features of human POV is that of expressing a "worldly sensitivity" (Hansen 266) visually characterized by the reversibility between the coupling of seeing/ seen: I'm seeing the world around me but l'm also seen simultaneously by others, and this reversibility (together with the reversibility between touching/touched) is what defines my being in the world, my embeddedness into an intersubjective world: "the seer is caught up in what he sees, it is still himself he sees" (Merleau-Ponty, The Visible and the Invisible 139). This coupling is molded 
on touching/touched, for which "I can identify the hand touched as the same one which will in a moment be touching [...]. The body [... tries] to touch itself while being touched and initiates a kind of 'reflection"' (Merleau-Ponty, Phenomenology of Perception 106).

The horizontal relation identified by Maurice Merleau-Ponty between seeing/ seen turns vertical once framed by the originary asymmetry between the eye and the gaze defined in the context of Lacanian psychoanalysis: "I'm seeing only from one point [an eye, or a POV], but in my existence I'm looked at [by the gaze] from all side" (Lacan 72). Cinema does something pretty interesting to these phenomenological and psycho-analytical categories: if it seems possible to say that cinema enforces the vertical relation between the eye and the gaze - the eye being the eye of the spectator and the gaze being the director's "all-seeing" (Lacan 75 ) - in the case of the cinematic technic of POV, eye and gaze collapse into each other. Thus, POV re-establishes the horizontal reversibility between seeing and seen (in this case between the seeing/seen of the viewer and director via the mediation of the actor). This reversibility of POV is at the root of its pharmacological nature, of its capacity of being both poison and cure.[5] In mainstream media, this capacity has been deployed to "disseminate ideology" and to enforce "consumer's behaviors" the way Adorno and Horkheimer explicate in their 1944 Dialectic of Enlightenment. The reversibility between eye and gaze produced by cinematic POV generates perceptive immersion and embodiment beyond the surface of the screen. The audience falls into the screen, embodied into the character's body, and the spectator's self-perception - understood as the state emerging from the affective gap the way it has been described in the above section III - can be directed by engineering the intertwining (Merleau-Ponty, The Visible and the Invisible
130-155) between seeing/seen — I'm seeing the actor/character moving in the scene and I'm seen (perceived) by myself as the very actor/character. This is the way in which POV-apparatus functions - by manipulating affects via perception. Thus, the immersivity produced by the figure of cinematic POV is harnessed to disseminate ideology and enforce consumer's behaviors. At the same time, the collapse between the eye and the gaze operated by cinematic POV re-arranges the relation between the coupling seeing/ seen and can produce the emergency of new forms of political agency. For example, POV mobile phone images recorded in the context of social unrest and protests and uploaded online (such as in the case of the so-called 'Arab Spring'), became the available format for revolutionary subjects to perform their political agency. The Egyptian Revolution has been an experimental ground for such grassroot emancipatory media practices.[6]

Finally, after having attempted to provide an understanding of the functioning of phenomenological POV and cinematic POV, I now turn to how the phenomenological intertwining of seeing/seen and eye/gaze is played out in the context of algorithmic POVs. This will be the focus of the last two sections of this article, where I will try to unpack the functioning of algorithmic POVs and their interaction with phenomenological POV and affects via two case studies: one referring to the "Arkangel" episode in the fourth series of Black Mirror, the other to the operationality of GANs (Generative Adversarial Networks).

\section{POV and algorithms}

"Now object perceives me" stated Paul Klee in his diaries, as cited by French philosopher Paul Virilio in the opening of his Vision Machine (1994), somehow prophetically 
envisioning a world of objects that learn how to see - and to "sense" - the surrounding space and the bodies occupying it. New technologies of vision oriented towards new forms of 'data-veillance' (Clarke 254-271) such as machine vision, seem to give technological consistency to Klee's intuition. Moreover, these technologies seem to confirm the asymmetry Lacan locates at the very heart of our phenomenological intertwining with the world, making visible the encompassing visual power of the (technological) gaze against the localized and punctual vision of the (human) eye. This a-symmetry is currently taking new forms that extend the capability of the gaze to all-seeing: for example, tracking technologies based on Al aim at quantifying a number of qualitative inputs that go from facial features and facial expressions to breathing pattern and heart beats - inputs that are the embodied manifestations of the affective patterns emerging from the affective gap. By quantifying them, algorithmic technologies points at accessing the very affective gap between action and reaction defining organic POV-matter - or its emergency as an affective, embodied POV. The technological gaze tries, thus, to vicariously access the eye by accessing the affective body right at the very moment where it emerges as a POV. In this sense, new technologies of vision based on $\mathrm{Al}$ - part of what Hansen calls $21^{\text {st }}$ century media - attempt to locate themselves at the very gap where the formation of a worldly sensitivity, or perception, emerges. Thus, algorithmic POV technologies invite us to re-think the notion of affect and Umwelt. In cinematic POV the overlapping between human and technology produces the overlapping between the regime of visibility of an embodied POV and the regime of visibility of the cinematic machine behind the surface of the screen: the audience feels inside the screen, inside the cinematic machine, and breaks through the so called fourth wall which technically separates the actors from the spectators.[7]

With algorithmic technologies the process looks similar but inverted. They produce the overlapping between the human and the machine by inserting the machine into the human, and not vice-versa, as in the case of cinema. To do so, they attempt to access human POV by accessing the very affective gap where it emerges from: first breaching through the screen of the body, and secondly extracting worldly data beyond the human conscious threshold. Thus, machines access vicariously a bodily dimension, while humans are exposed to a quantified version of their very affective fabric, which - datafied - contributes to the constitution of new forms of human-machinic Umwelten with complex political implications. One of the most significant change in relation to these new forms of Umwelt, consists in the fact that the affordances between the human and the surrounding space are technically anticipated by a capture which re-defines affordances as such and which claims to design them in ways that fulfil the subject's expectations better than the subject's agency could possibly achieve. This is what happens in relation to the creation of POV data-doubles and the consequent formation of filter bubbles within social networks based on the anticipation of users' affects[8] emerging from the affective gap. "Anticipation made possible by algorithms [...] become increasingly active, to the point of displacing or marginalizing active directedness" (Hui 144). Algorithmic POVs manufacture an "automatic future, in which our selections will be to a large extent, if not completely, predefined according to a specific schema and index" (Hui 150). This is what happens in "Arkangel" directed by Jody Foster - the second episode of the fourth season of Black Mirror - where a mother implants her daughter with a device which allows her to see what she is looking at from 
a POV perspective in real-time. The mother is provided with a user-friendly interface that gives her the possibility of deleting images from her daughter's sight stream that she thinks could traumatize her - such as images of conflicts or violence. As a consequence of this technology, the daughter grows incapable of recognizing conflicts or violence and indeed becomes incapable of behaving appropriately in such circumstances. In "Arkangel", algorithms implement a realitybubble around children, one perceptively different from the material reality lived by others. In this example, the direct capturing of affects by algorithmic technologies goes beyond perception in the sense that perception appears here as a malleable context to fully engineer, to anticipate and design affects.

These algorithmic technologies attempt to anticipate the affordances that define the relation between organic POVs and their Umwelten by designing POV-data doubles retro-actively producing the affective subjects they're generated from. Algorithmic POV handles the relation between seeing/seen by directly harnessing the gap between the two: anticipating the subject's affordances and projecting back into the subject an algorithmic POV data-double which informs the way the subject operates within her own Umwelt. This is the only way algorithmic POV technologies can currently and vicariously access affects despite their incapability of producing the gap at the core of organic POVs. In doing so, the (algorithmic) gaze attempts to access the (phenomenological) eye and to control it by vicariously accessing the affective gap, and bypassing perception.

\section{Towards a phenomeno- logical understanding of GANs}

Are there any other ways in which algorithmic POVs attempt to reproduce the affective gap of phenomenological POVs? The operationality of GANs provides an exemplary case study of new forms in which algorithmic POVs try to approximate the functioning of phenomenological POVs. GANs mimic the very intertwining between seeing/seen and eye/gaze to which Merleau-Ponty and Lacan refer when pointing to the enworlding of phenomenological POVs. GANs are the most advanced form of algorithmic simulation of a phenomenological enworlding, as I hope to illustrate during the course of this last section. What exactly is a GAN, and why am I saying that GANs provide an example of a new radical way to attempt the simulation of the affective gap at the core of phenomenological POV?

GANs are a form of unsupervised machine learning able to access raw data from the world and to build an understanding of them without the mediation of any linguistic labeling applied by humans, or "mechanical turks" (Wikipedia), which tag huge datasets of images and prepare them to train "supervised machine learning algorithms" (Wikipedia). GANs build an understanding of raw data by establishing an antagonistic relation between two neural networks, one generating data (generator), the other discriminating the data generated on the basis of a model (discriminator).

In a sense, generator and discriminator constitute each other through an algorithmic exchange that closely resembles both the intertwining of the coupling seeing/seen and the split between the eye and the gaze. Generator and discriminator see each other 
and in doing so establish each other's (inorganic and algorithmic) POV, while at the same time enacting the distinctive roles of the eye (generator) and of the gaze (discriminator). At the same time, a form of technological Umwelt appears as the place of the emergency of GANs' affordances. GANs' Umwelt emerges at the intersection of generator and discriminator's affordances, and takes the form of what is technically addressed as latent space. Latent space is defined as the space where a "generative network learns to map [...] a particular data distribution of interest, while the discriminative network discriminates between instances from the true data distribution and candidates produced by the generator" (Wikipedia). The latent space can be addressed as an algorithmic screen where an algorithmic recognition happens a recognition based on the interplay between generator and discriminator's different algorithmic POVs. This algorithmic recognition produces a form of algorithmic perception where the complexity of the intertwining between embodied POVs is reduced to a task-oriented statistical capability of pattern recognition - a feature typical of $\mathrm{Al}$ in general, according to Matteo Pasquinelli (2017). The latent space is the algorithmic screen where a form of algorithmic gap emerges between the images produced by the generator, and the model on which the discriminator has been calibrated, when generator and discriminator's POVs mismatch. There's no affect in this gap which emerges with the emergencies of the intertwining between generator and discriminator's mismatching POVs. Furthermore, the gap is filled once the generator and discriminator's POV perfectly overlap - which happens when the generator produces a closer enough version of the model capable of fooling the discriminator. In this algorithmic intertwining, subject and object coincide when the gap is closed, not when it appears such as in the case of organic
POV-matter. In brief, the phenomenological intertwining between organic POV-matter(s) is rooted in the affective gap from where selfperception - understood as the coincidence between subject and object - emerges. The intertwining between generator and discriminator in a GAN, in contrast, is rooted in the algorithmic gap emerging from the mismatching between generator and discriminator's POVs. In other words, if in phenomenological POV the coincidence between subject and object emerges from the affective gap, in GANs the coincidence between subject and object happens when the gap is closed and generator and discriminator POV coincides. This happens when the generator generates an image which fools the discriminator and closely matches the model.

If, according to Bergson, there's no perception without affection, when it comes to GANs and algorithmic POVs in general, we can only metaphorically refer to perception - as much as to POVs and Umwelt. Nevertheless, GANs re-invent the relation between POV, affects and Umwelt, and do so by deploying algorithms that mimic the phenomenological intertwining that is characteristic of embodied POV. If GANs reproduce the phenomenological intertwining between seeing/seen and eye/gaze, other types of algorithmic POVs access the body by harnessing the affective gap from within, constituting POV data-doubles and retro-projecting them on the affective subject they have been generated from, bypassing perception (as in the case of Arkangel). These forms of intensive or molecular algorithmic capture of the affective gap, differ from the functioning of earlier media which, instead, operate at a molar level, and subsume affection into perception. Cinematic POV does so by collapsing the eye of the audience and the gaze of the director via the body of the actor-character. The common feature between cinematic and algorithmic POV consists in the shrinking of 
the distance between the eye and the gaze. This shrinking happens in parallel to the shrinking between inorganic POV-matter and organic POV-matter. Once the former turns into technological inorganic POV-matter, it turns into a POV-apparatus which tends to operationalize the reduction of the distance between the interface and the body, and as a consequence between the (technological) gaze and the (phenomenological) eye. This is the common feature between cinematic POV and algorithmic POV, despite the fact that they operate this reduction differently, the former subsuming affection via perception, the latter subsuming affection directly, bypassing perception. By doing so, the POV-apparatus produces both new regimes of visibility with related affects and Umwelten - and new regimes of truth. From the differences between a phenomenological, cinematic and algorithmic form of POV, my argument moves towards defining the techno-phenomenological conditions for the emergency of both new regimes of '(post-)truth',[9] and a new form of visual governmentality, which I refer to as 'POV-opticon'.[10]

\section{Notes}

[1] 'Pre-biotic soup' is an expression related to the unstable state of matter in which chemical compounds were about to generate the conditions for life over the planet Earth at a certain stage of its evolution (CERN).

[2] Spins describe electrons spinning around nuclei of protons and neutrons forming the first atoms some 380,000 years after the Big Bang (Cern.com).

[3] The 'tick' is a famous example emphasized by both Uexküll and Deleuze. See Uexküll's A Foray into the Worlds of Animals and Humans, and Deleuze's L'Abécédaire de Gilles Deleuze.

[4] See Stiegler's Technics and Time I. The Fault of Epimetheus.

[5] The pharmacological nature of technology is highlighted by Bernard Stiegler throughout his philosophy. See Stiegler's "Pharmacology of Desire".

[6] I have been writing about media activism in Egypt during the revolution while being based in Cairo and collaborating with activist collectives. See Azar,"The Revolution will not be Tweeted (?)".

[7] The 'fourth wall' mirrors the a-symmetry Lacan refers to the relation between the eye and the gaze: if the spectator can see the actors behind the fourth wall, the actors act without seeing behind the fourth wall that somebody is seeing them. And yet the seeing of the spectator is driven by the seeing of the actors. 
[8] I have previously explored this topic in relation to the production of a new type of selfie aesthetic in a paper published last year in APRJA; see Azar's "The Algorithmic Facial Image (AFI) and the relation between truth value and money value". Another example of these forms of prehension is a new MIT prototype that allow users to control basic functions of a computer through an ergonomic wearable interface able to record the micro-movements of the subject's lower jaw as a way to infer brain activity - the jaw moves slightly when the brain formulate a decision even without the production of a verbal utterance - and before the awareness of the subject: "Electrodes on the face and jaw pick up otherwise undetectable neuromuscular signals triggered by internal verbalizations". See Herdesty's "Computer system transcribes words users 'speak silently"'.

[9] See Azar's "The Algorithmic Facial Image $(\mathrm{AFI})$ and the relation between truth value and money value". The paper is forthcoming as a chapter of G. Lovink and D. Della Ratta (eds), Online Self. Palgrave and McMillan, 2021.

[10] I've started investigating the relation between POV, regimes of truth and games of truth in a paper presented at the 2018 After Post-Truth conference in Barcelona. See Azar, "From Panopticon to POVopticon: drive to visibility and games of truth" (draft version of the paper can be found on academia.edu).

\section{Works cited}

Adorno, Theodor, and Max Horkheimer. Dialectic of Enlightment. Continuum, 1993.

Azar, Mitra. "From Panopticon to POVopticon: drive to visibility and games of truth". Academia.edu, https://www. academia.edu/37648284/From_Panopticon_ to_POV-opticon_drive_to_visibility_and_ games_of_truth.

Azar, Mitra. "The Algorithmic Facial Image (AFI) and the Relation between Truth Value and Money Value." APRJA, 8.1, June 2018, http://www.aprja.net/ the-algorithmic-facial-image/.

Azar, Mitra. "The Revolution will not be Tweeted (?): Media-attivismo al Cairo durante la post-rivoluzione." Alfabeta, May 2012.

Bergson, Henri. Matter and Memory. George Allen and Unwin, 1911.

Braningan, Edward. POV in The Cinema. A Theory of Narration and Subjectivity in Classical Film. De Gruyter Mouton, 1984.

Clarke, Roger. "Information Technology and Dataveillance". Commun. ACM 31, 5, 1988, pp. 498-512.

Deleuze, Gilles. Cinéma 1. The MovementImage. University of Minnesota Press, 1986.

Emmeche, C. "Can robots have an Umwelt". Semiotica vol. 134 (issue 1/4), 2001, pp. 653-693. 
Deleuze, Gilles. L'Abécédaire de Gilles

Deleuze, by Pierre-André Boutang, interview with Claire Parnet, Éditions Montparnasse, 2004.

Foster, Jodi, Dir. "Arkangel," 2nd episode, Black Mirror series, 4th season. Netflix, 1997.

Herdesty, Larry. "Computer system transcribes words users speak silently". MIT News Office, http://news.mit.edu/2018/ computer-system-transcribes-words-usersspeak-silently-0404.

Hansen, Mark B. N. Feed-forward. University of Chicago Press, 2015.

Hansen, Mark B. N. New Philosophy for New Media. MIT Press, 2004.

Lacan, Jacques. The Four Fundamental Concepts of Psycho-analysis. Routledge (1973) [2018].

Massumi, Brian. "The Autonomy of Affect". Cultural Critique, No. 31, The Politics of Systems and Environments, Part II. Autumn, 1995, pp. 83-109.

Mechanical Turks. Wikipedia. https:// en.wikipedia.org/wiki/Mechanical_Turk.

Merleau-Ponty, Maurice. Phenomenology of Perception. Routledge, 2002.

Merleau-Ponty, Maurice. Eye and Mind: Merleau-Ponty Reader, edited by Toadvine, T. Lawlor, L. Northwestern University Press, 2009.

Merleau-Ponty, Maurice. The Visible and the Invisible. Northwestern University Press, 1968. Print.
Pasquinelli, Matteo. "Machines that Morph Logic: Neural Networks and the Distorted Automation of Intelligence as Statistical Inference". Glass Bead, 1: "Logic Gate: The Politics of the Artifactual Mind", 2017. Ponsoldt, James. The Circle. STXfilms, Europacorp, 2017.

Spin. Cern.com, https://home.cern/science/ physics/early-universe.

Stiegler, Bernard. Technics and Time I: The Fault of Epimetheus. Stanford University Press, 1998.

Stiegler, Bernard. "Pharmacology of Desire: Drive-based Capitalism and Libidinal Dis-economy." New Formations 72, 2011, pp. 150-161.

Supervised Learning, Wikipedia. https:// en.wikipedia.org/wiki/Supervised_learning.

The Early Universe, Cern.com, https:// home.cern/science/physics/early-universe.

Uexküll, Jacob V. "The Theory of Meaning." Semiotica 42(1): 25-82 1982.

Uexküll, Jacob V. A Foray into the Worlds of Animals and Humans. University of Minnesotta Press, 2010.

Uexküll, Jacob V. "Die Bedeutung der Umweltforschung für die Erkenntnis des Lebens." Zeitschrift für die gesamte Naturwissenschaft 1.7, 1935, pp. 257-272.

Virilio, Paul. The Vision Machine. University of Indiana Press, 1994.

Wittgenstein, Ludwig. Philosophical Grammar. Blackwell, 1974. 